\title{
Development of Superconducting Wind Turbine Generators
}

\author{
Jensen, Bogi Bech; Mijatovic, Nenad; Abrahamsen, Asger Bech
}

Published in:

Scientific Proceedings of EWEA 2012 - European Wind Energy Conference \& Exhibition

Publication date:

2012

Document Version

Publisher's PDF, also known as Version of record

Link back to DTU Orbit

Citation (APA):

Jensen, B. B., Mijatovic, N., \& Abrahamsen, A. B. (2012). Development of Superconducting Wind Turbine Generators. In Scientific Proceedings of EWEA 2012 - European Wind Energy Conference \& Exhibition European Wind Energy Association (EWEA).

\section{General rights}

Copyright and moral rights for the publications made accessible in the public portal are retained by the authors and/or other copyright owners and it is a condition of accessing publications that users recognise and abide by the legal requirements associated with these rights.

- Users may download and print one copy of any publication from the public portal for the purpose of private study or research.

- You may not further distribute the material or use it for any profit-making activity or commercial gain

- You may freely distribute the URL identifying the publication in the public portal

If you believe that this document breaches copyright please contact us providing details, and we will remove access to the work immediately and investigate your claim. 


\section{Development of Superconducting Wind Turbine Generators}

Bogi B. Jensen ${ }^{1}$, Nenad Mijatovic ${ }^{1}$, Asger B. Abrahamsen ${ }^{2}$

${ }^{1}$ Department of Electrical Engineering, Technical University of Denmark, Denmark

2 Department of Wind Energy, Technical University of Denmark, Denmark

18-04-2012

EWEA 2012 Copenhagen, Denmark

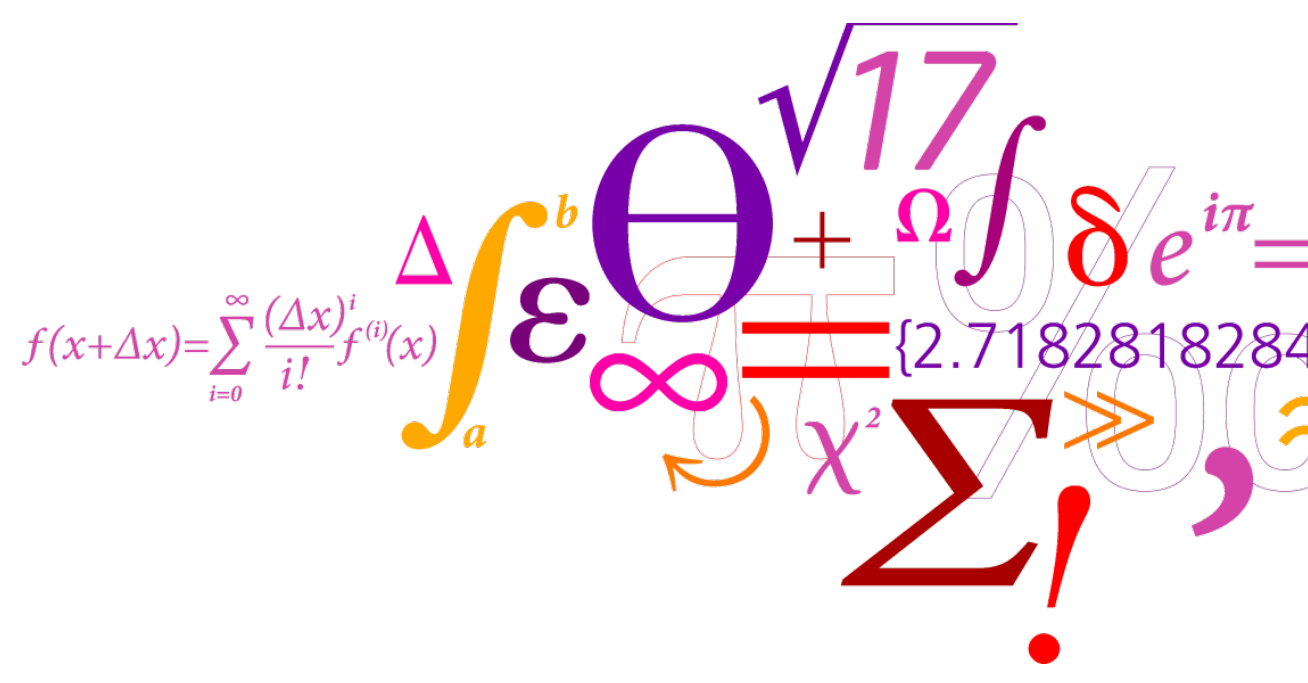

DTU Electrical Engineering

Department of Electrical Engineering 


\section{Development of wind turbines}

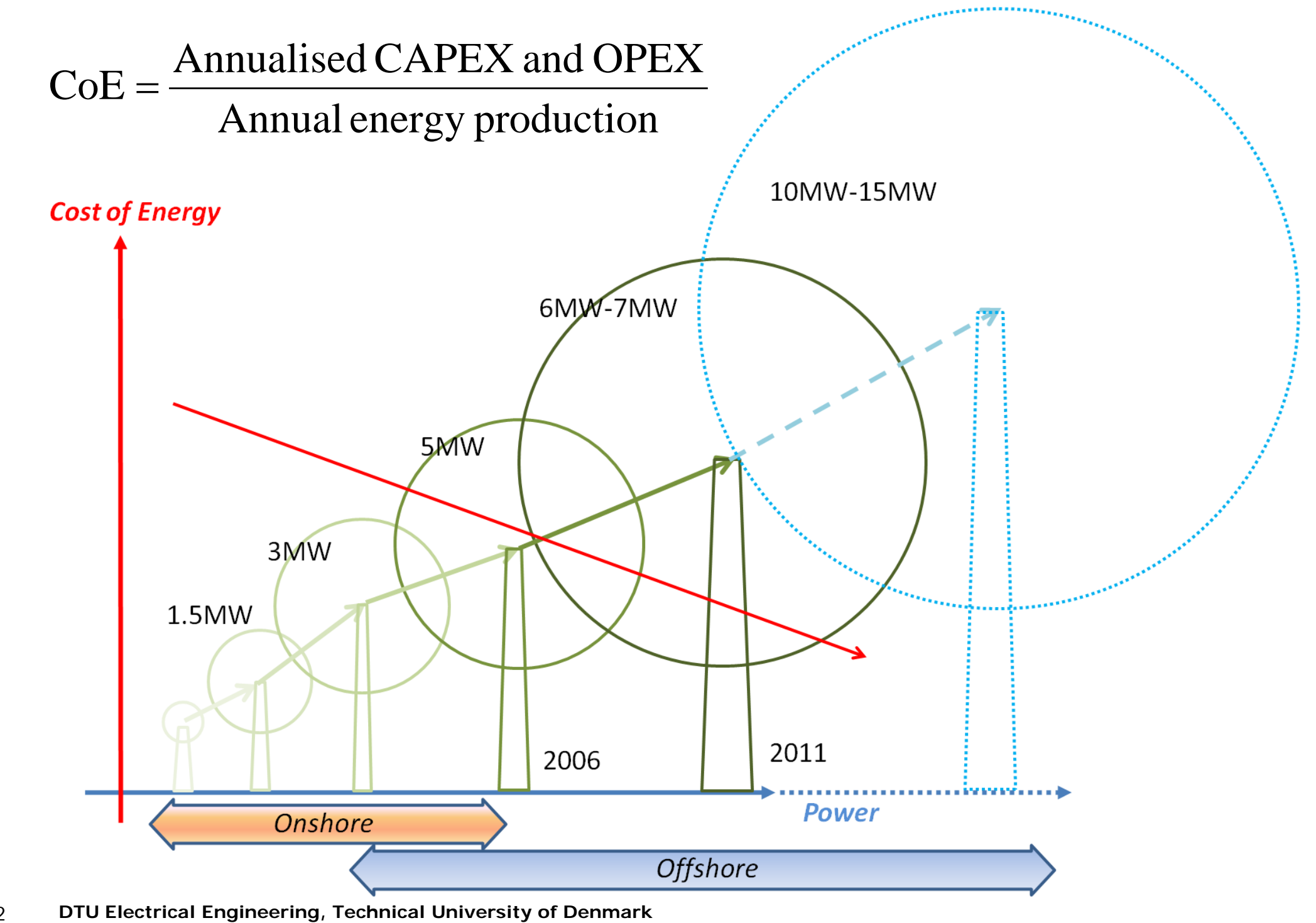




\section{$5 \mathrm{MW}$ and beyond}

\begin{tabular}{l|l|l|}
\hline Manufacturer & Transmission & Generator \\
\hline Siemens Wind Power & Direct drive & PMSG 6.0MW \\
\hline Vestas & Medium speed & PMSG 7.0MW \\
\hline Enercon & Direct drive & EESG 7.5MW \\
\hline Alstom & Direct drive & PMSG 6.0MW \\
\hline REPower & High speed & DFIG 6.2MW \\
\hline Areva & Low speed & PMSG 5.0MW \\
\hline $\mathbf{1 O M W}$ and beyond & - proposals/ investigations \\
\hline Manufacturer & Transmission & Generator \\
\hline American Superconductor & Direct drive & HTS 10MW \\
\hline General Electric & Direct drive & LTS 10-15MW \\
\hline Advanced Magnet Lab & Direct drive & MgB 2 10MW
\end{tabular}




\section{Schematic of a Superconducting Machine}

- The superconductor must be kept at cryogenic temperatures

- The armature winding is usually proposed to be copper at ambient temperature

$$
\begin{aligned}
& P=\omega \times T \\
& T \propto A \times B \times V \\
& P: \text { power } \\
& T: \text { torque } \\
& \omega: \text { rotational speed } \\
& A: \text { electric loading } \\
& B: \text { magnetic loading } \\
& V: \text { volume }
\end{aligned}
$$
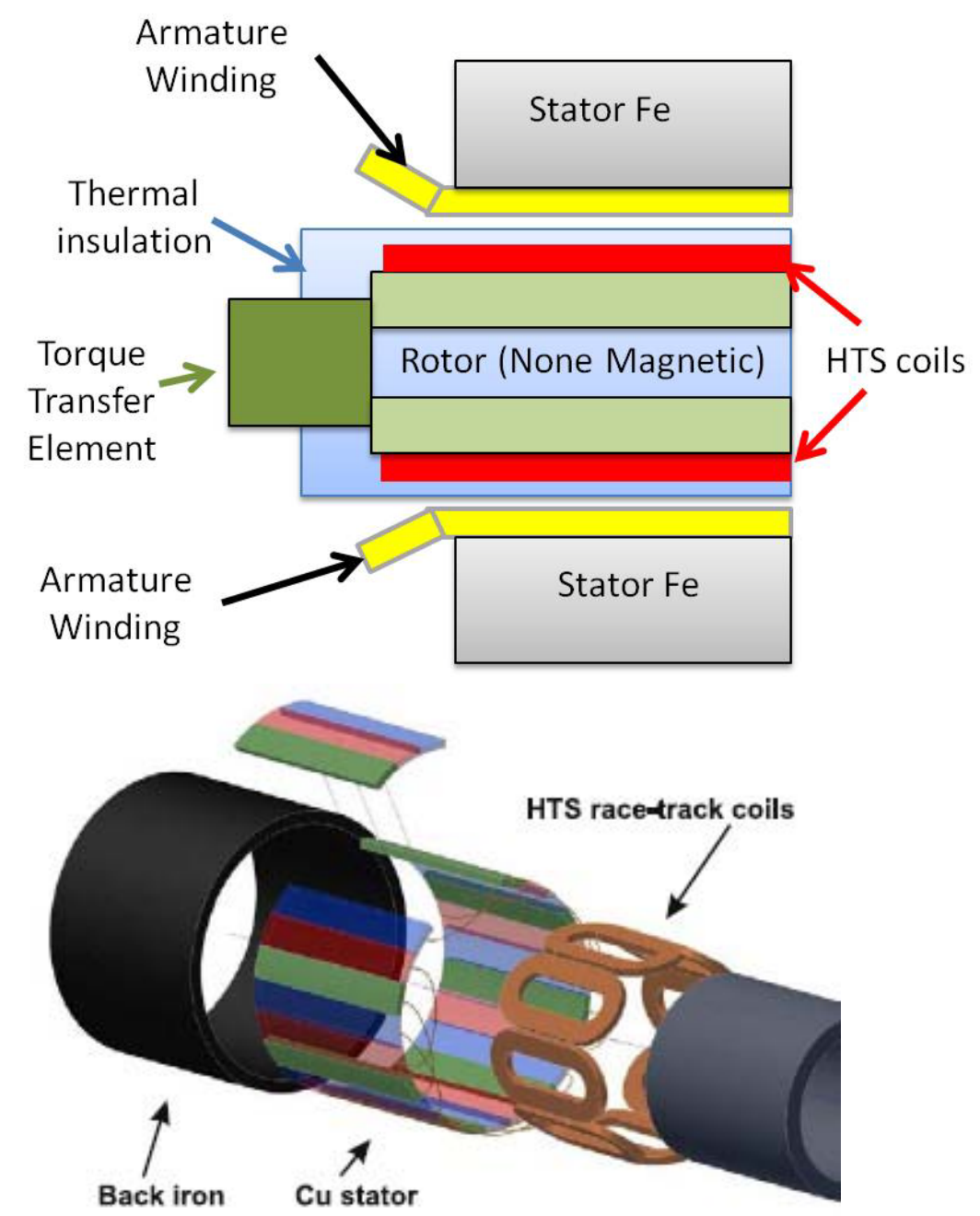


\section{High Temperature Superconductors}

- The superconducting state is limited by

- Critical flux density $B_{c}$

- Critical current density $J_{c}$

- Critical temperature $T_{c}$
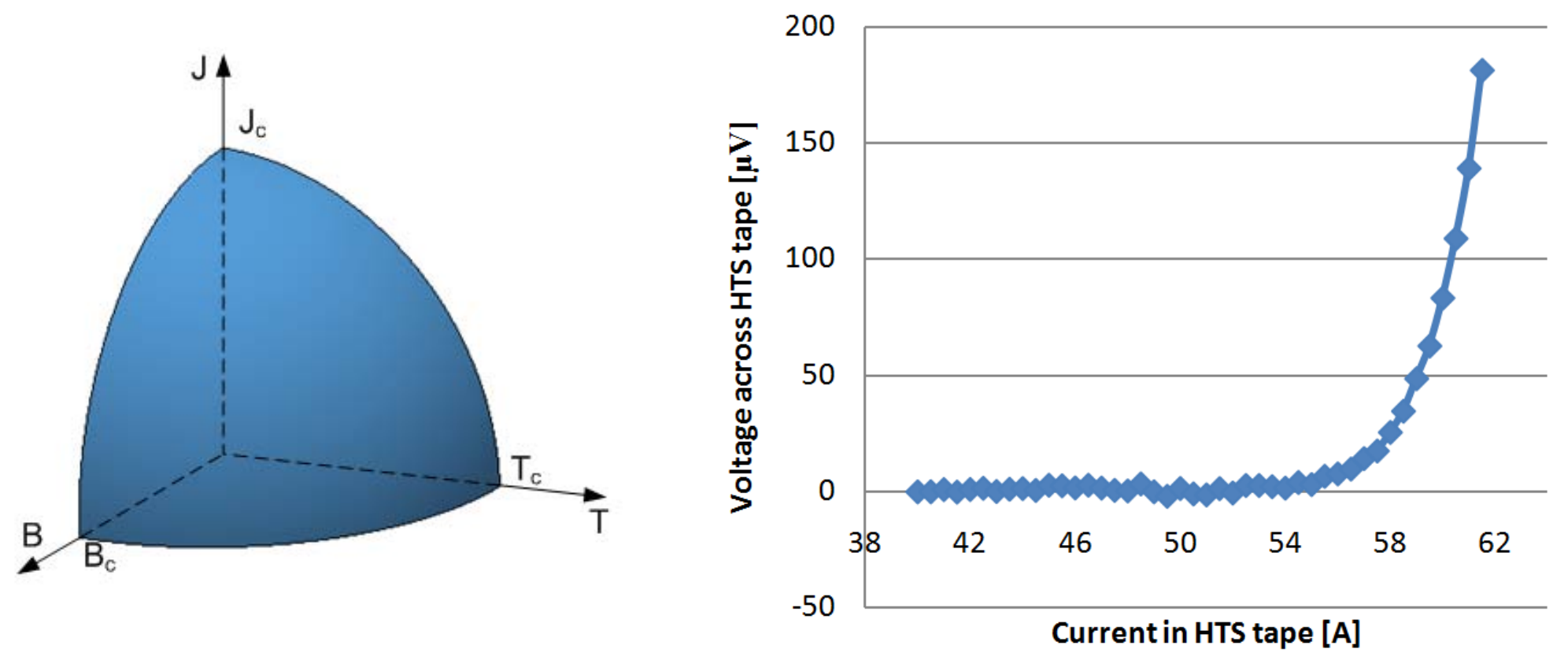


\section{Overview of superconductors}

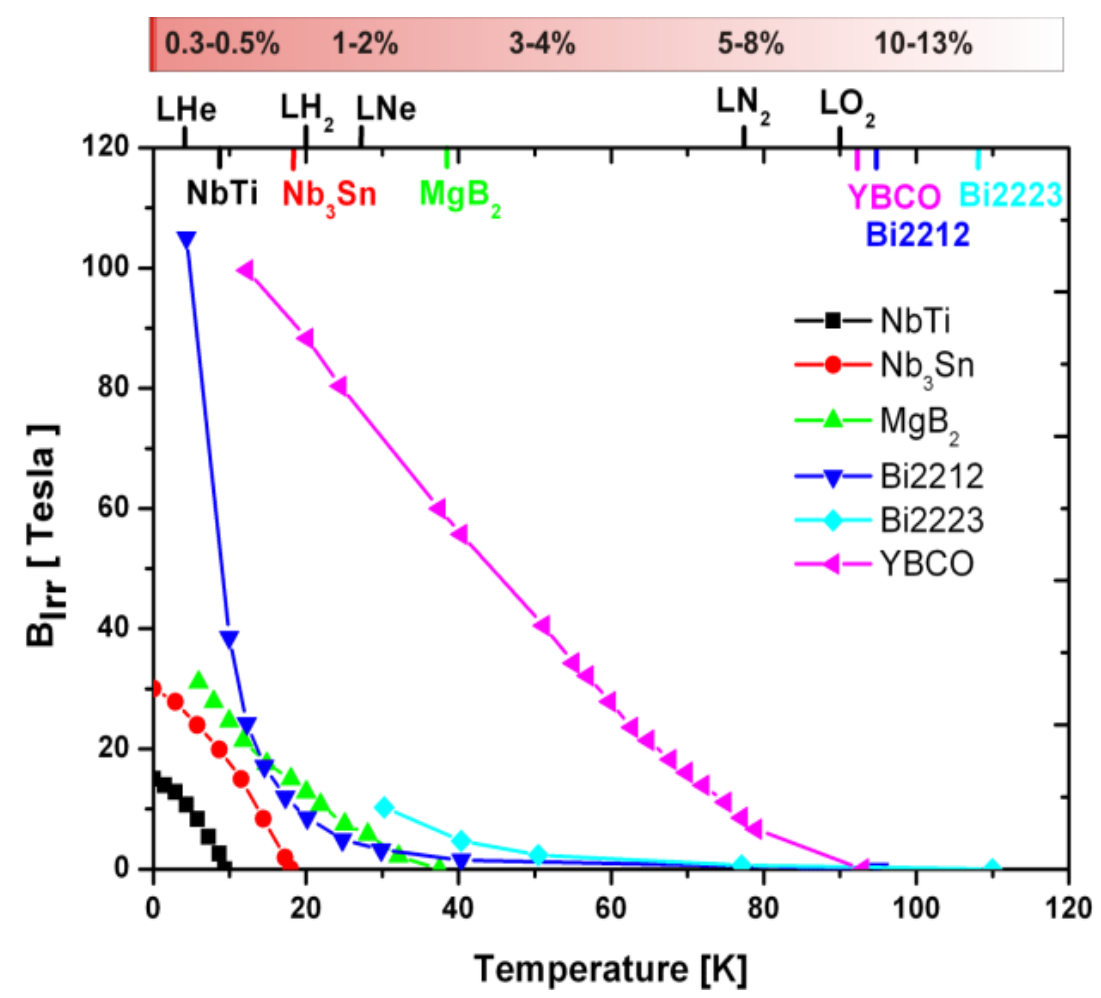

\section{Metal strip}

\begin{tabular}{|c|c|c|c|c|}
\hline Type & $\begin{array}{l}\text { Price } \\
€ / m\end{array}$ & $\begin{array}{l}\mathrm{J} \\
\mathrm{A} / \mathrm{mm}^{2}\end{array}$ & $\begin{array}{l}\text { Flux } \\
\text { density [T] }\end{array}$ & $\begin{array}{l}\text { Temp. } \\
{[\mathrm{K}]}\end{array}$ \\
\hline $\mathrm{NbTi}$ & 0.4 & $10^{3}$ & 5 & 4.2 \\
\hline $\mathrm{Nb}_{3} \mathrm{Sn}$ & 3 & $1-4 \times 10^{3}$ & 5 & 4.2 \\
\hline $\mathrm{MgB}_{2}$ & 4 & $10^{2}$ & 3 & 20 \\
\hline $\mathrm{Bi}-2223$ & 20 & $\begin{array}{l}390 \\
10\end{array}$ & $\begin{array}{l}3 \perp \text { tape } \\
3 \perp \text { tape }\end{array}$ & $\begin{array}{l}20 \\
50\end{array}$ \\
\hline YBCO & 30 & $\begin{array}{l}98(480) \\
49(190)\end{array}$ & $\begin{array}{l}3 \perp \text { tape } \\
3 \perp \text { tape }\end{array}$ & $\begin{array}{l}20 \\
50\end{array}$ \\
\hline
\end{tabular}




\section{Cooling system}

- The superconductors need to be cold:

$<5 \mathrm{~K}$ for LTS

$<20 \mathrm{~K}$ for $\mathrm{MgB}_{2}$

30-50K for HTS

- Insulation requires large effective airgap

- Large fault currents and torques

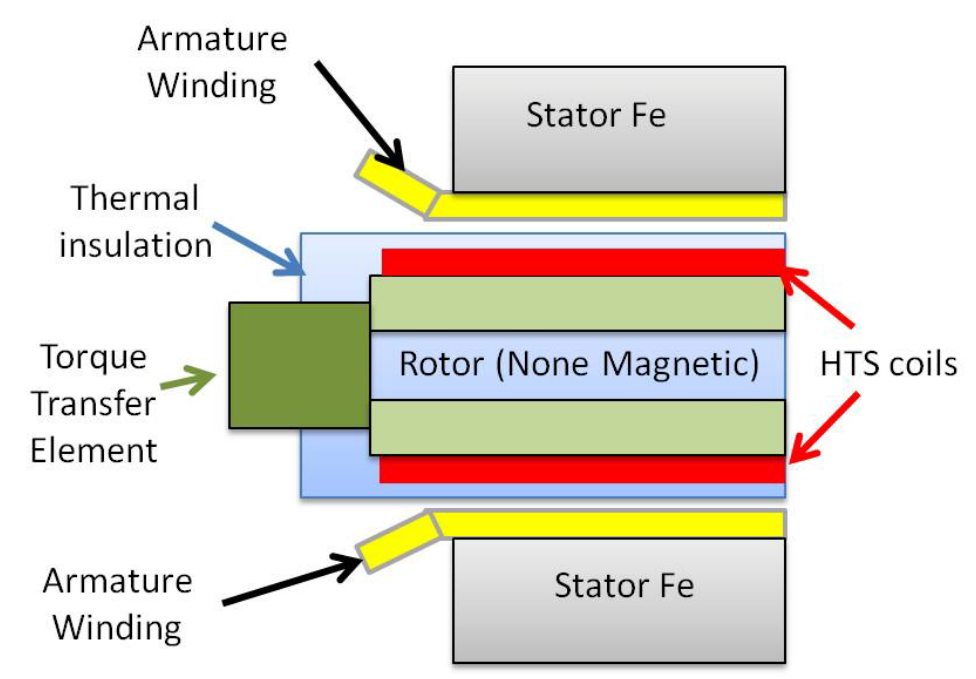

- Torque transfer

- Reliability has yet to be proven and requires years of operating experience

- Production capacity of HTS and $\mathrm{MgB}_{2}$ are currently not adequate for largescale commercialisation - this should change if the need is present 


\section{Advantages}

- Very high torque density

$$
P=\omega \times T \quad, \quad T \propto A \times B \times V
$$

- Higher efficiency than an equivalent direct drive PM generator

$$
P_{\mathrm{Cu}}=I_{\mathrm{Cu}}{ }^{2} R_{\mathrm{Cu}}=J_{\mathrm{Cu}}{ }^{2} A_{\mathrm{Cu}}{ }^{2} \frac{l_{\mathrm{Cu}}}{A_{\mathrm{Cu}} \sigma_{\mathrm{Cu}}}=\frac{J_{\mathrm{Cu}}{ }^{2} V_{\mathrm{Cu}}}{\sigma_{\mathrm{Cu}}}
$$

- Very limited dependence on rare earth materials

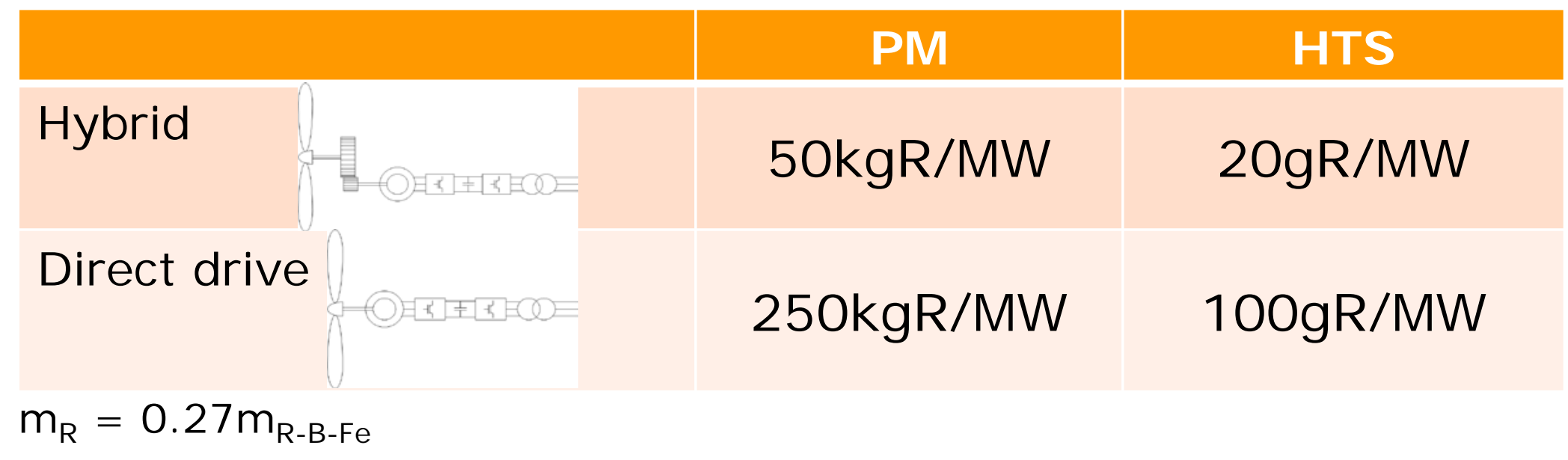




\section{American Superconductor (AMSC) SeaTitan 10MW}

- HTS - Superconducting field winding

- Copper armature winding

- Generator diameter: 4.5-5 meters

- Weight: 150-180 tonnes (55-66 Nm/kg)

- Efficiency at rated load: $96 \%$

Highest torque HTS machine intended for ship propulsion:

-36.5MW @ 120rpm

-2.9MNm @ 75 tons

- $39 \mathrm{Nm} / \mathrm{kg}$

- Challenge

- HTS price and availability

- Advantage

- Relatively simple cooling system with off-the-shelf solutions

- Cooling power

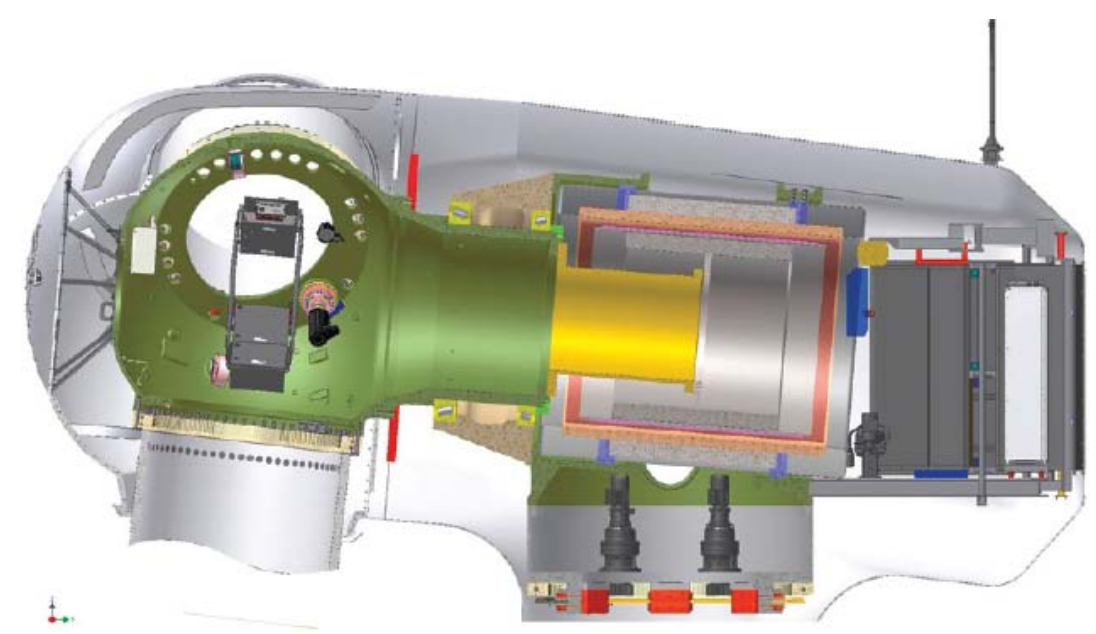

Reproduced with permission from AMSC 


\section{General Electric (GE) 10-15MW}

- LTS - Superconducting field winding

- Extensive experience from the MRI sector

- Rotating armature

- Challenge

- Complicated cooling system and higher cooling power

- Advantage

- Proven technology from MRI

- Cheaper superconductor

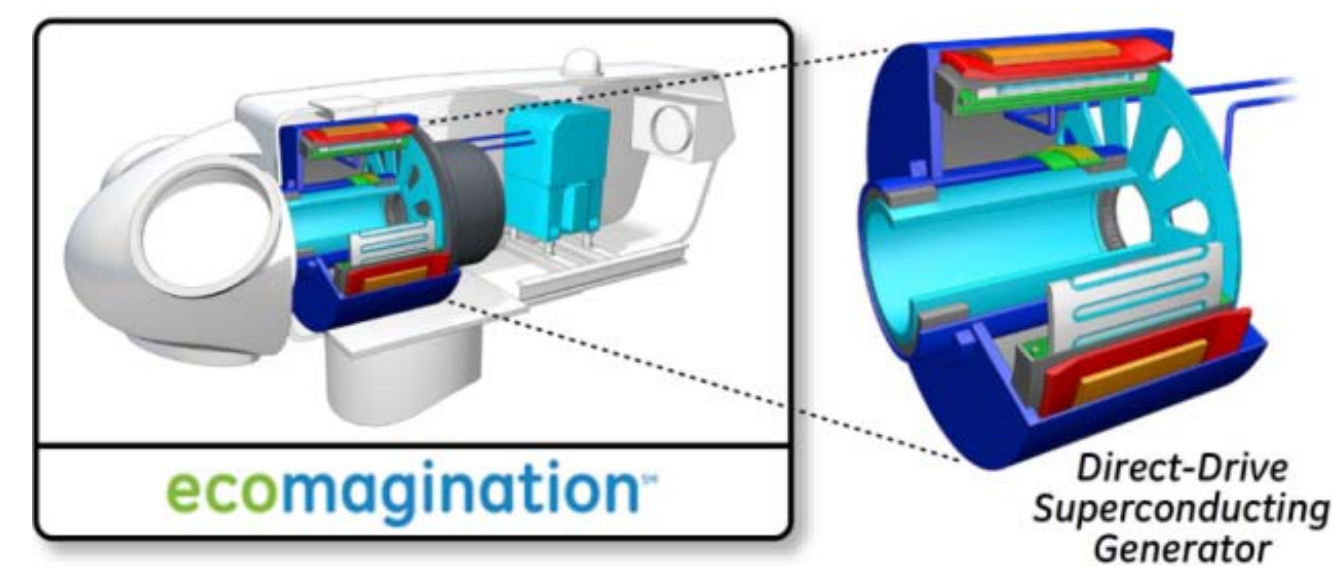

Reproduced with permission from GE 


\section{Advanced Magnet Lab (AML) $10 \mathrm{MW}$ fully superconducting}

- $\mathrm{MgB}_{2}$ - Fully superconducting generator

- Superconducting field winding

- Superconducting armature winding

- Challenge

- Complicated cooling system and higher cooling power

- I mprovement in $\mathrm{MgB}_{2}$ wire is needed

- Advantage

- Cheap superconductor

- Fully superconducting

- More torque dense

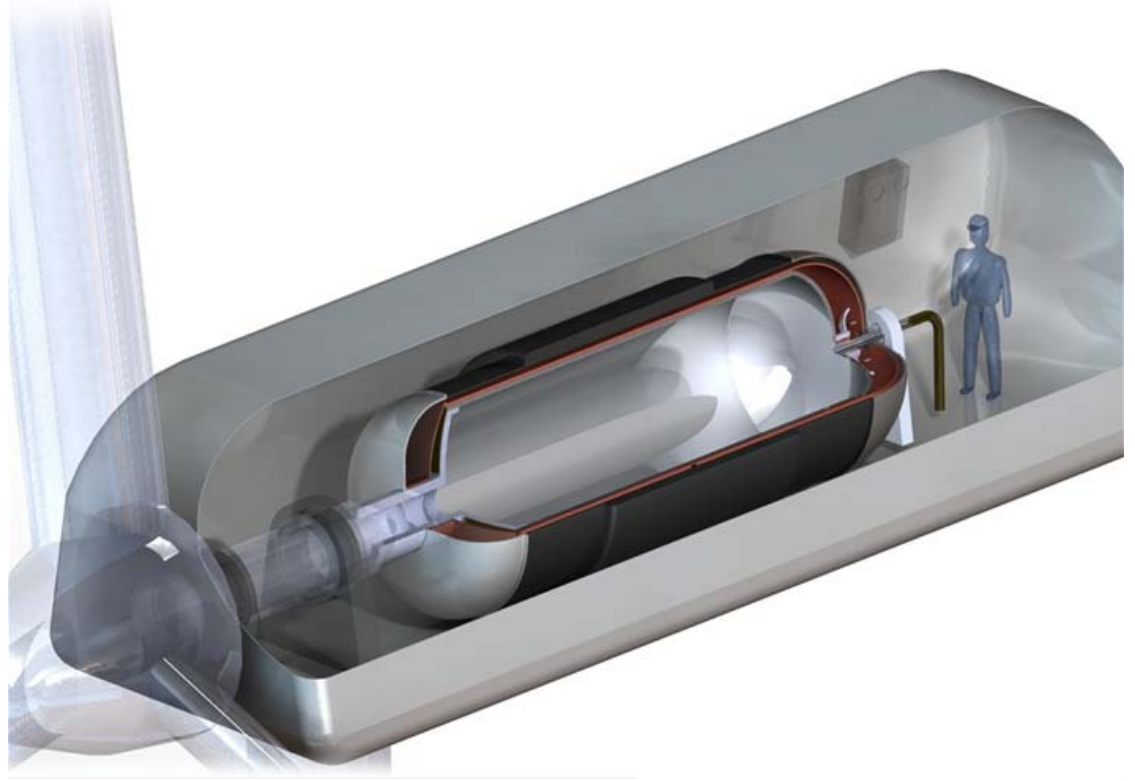

Reproduced with permission from AML

$$
P=\omega \times T \quad, \quad T \propto A \times B \times V
$$




\section{Patent Development}

-Web of Knowledge search with keywords: "Supercond*" and "machin*"

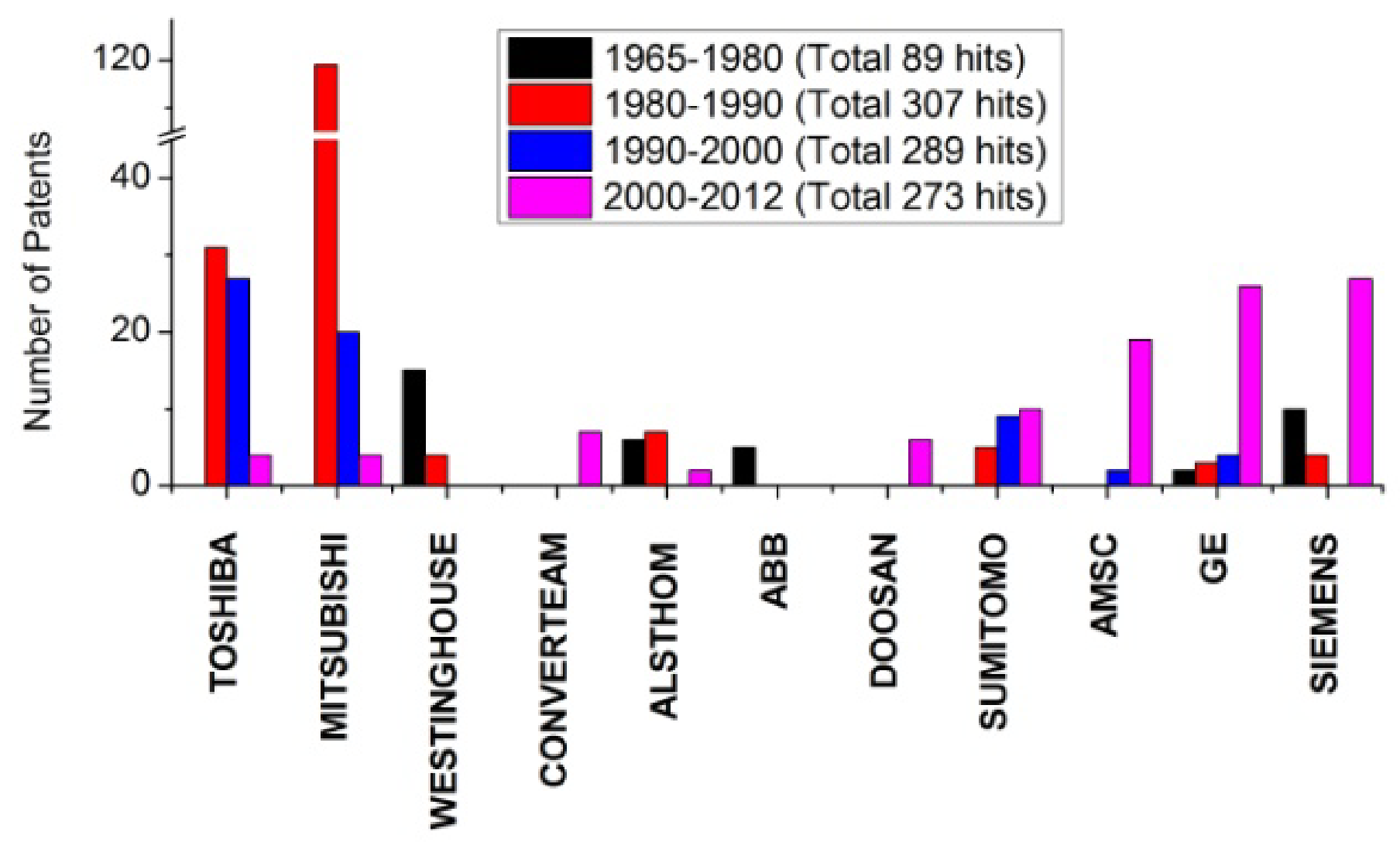




\section{Discussion and conclusion}

- Superconducting generators might be the answer to large wind turbines

- Smaller generator

- Less RE demand by a three orders of magnitude

- A collaborative effort is needed including:

- Wire manufacturers

- Wind turbine manufacturers

- Wind turbine operators

- Large-scale demonstrators are needed

- To test the performance in a wind turbine

- To test the reliability

- Thank you 